\title{
AN ORDER-THEORETIC DESCRIPTION OF MARINESCU SPACES
}

\author{
W. A. FELDMAN AND J. F. PORTER
}

\begin{abstract}
It is shown that any inductive limit $E$ in the category of convergence spaces of real locally convex topological vector spaces (i.e., any Marinescu space) can be embedded in a partially ordered vector space so that convergence in $E$ can be characterized as an order-theoretic convergence. The ordertheoretic convergence in question is a modification of classical order convergence.
\end{abstract}

Introduction. DeMarr [1] has shown that every real Hausdorff locally convex space $V$ can be embedded in a partially ordered vector space so that the convergence in $V$ can be realized as unbounded order convergence. In the theorem of $\$ 2$ we provide an analogous order-theoretic description of real Marinescu spaces. Marinescu spaces include locally convex spaces (not necessarily Hausdorff) as well as important examples of nontopological convergence vector spaces; pertinent examples can be found in [2] and [3]. It is of interest to note that the theorem provides an order-theoretic characterization of almost-everywhere convergence of nets of measurable functions on $[0,1]$ (see $\S 1)$. This nontopological convergence, when restricted to sequences, has previously been described order-theoretically (e.g., see [1] and [4]), but these characterizations do not extend to arbitrary nets.

1. Preliminaries. Throughout this paper we restrict ourselves to vector spaces over the reals. We begin by reviewing the definition of a Marinescu space (see [2]). Let $E$ be a vector space and $\left\{E_{\theta}\right\}_{\theta \in \sigma}$ ( $\sigma$ some index set) a collection of subspaces of $E$ satisfying:

1. $\bigcup_{\theta \in \sigma} E_{\theta}=E$.

2. Each $E_{\theta}(\theta \in \sigma)$ is a locally convex space (not necessarily Hausdorff).

3. If $\theta^{\prime}$ and $\theta^{\prime \prime}$ are in $\sigma$ there is a $\theta$ in $\sigma$ such that $E_{\theta^{\prime}} \cup E_{\theta^{*}} \subseteq E_{\theta}$ and the topologies on $E_{\theta^{\prime}}$ and $E_{\theta^{\prime \prime}}$ are finer than those induced by $E_{\theta}$.

Presented to the Society, January 26, 1973; received by the editors January 15, 1973.

AMS (MOS) subject classifications (1970). Primary 54A05, 06A65; Secondary 54A05, 46A40.

Key words and phrases. Convergence space, Marineşcu space, partial order, ordertheoretic convergence.

(c) American Mathematical Society 1973 
The inductive limit in the category of convergence spaces of this system $\left\{E_{\theta}\right\}_{\theta \in \sigma}$ is called a Marinescu space, which we again denote by $E$.

We will utilize the following characterization of convergence in $E$ (immediate from the definition):

A net $\left\{x_{\alpha}\right\}$ in $E$ converges to a point $x$ in $E$ if and only if there is a subspace $E_{\theta}$ containing $x$ such that $\left\{x_{\alpha}\right\}$ is eventually in every neighborhood of $x$ in $E_{\theta}$.

To show that almost-everywhere convergence can be viewed in the context of Marinescu spaces, we let $S[0,1]$ denote the space of measurable functions on $[0,1]$, together with almost-everywhere convergence. Let $\mathscr{U}$ be the family of subsets of $[0,1]$ having measure zero. For each $U \in \mathscr{U}$ we let $S_{U}$ denote the measurable functions on $[0,1]$ together with pointwise convergence on the complement of $U$. Each $S_{U}$ is a non-Hausdorff locally convex space and $S[0,1]$ is the inductive limit of the system $\left\{S_{U}\right\}_{U \in \mathscr{U}}$.

The remainder of this section is devoted to introducing the ordertheoretic concepts necessary for this paper.

Let $(X, P)$ be a partially ordered vector space with positive cone $P$ (i.e. $P+P \subseteq P, \lambda P \subseteq P$ for $\lambda \geqq 0$ and $P \cap(-P)=\{0\}$ ). We denote by $P^{*}$ the set $P \backslash\{0\}$. A set $M \subseteq P$ is said to be directed downward if for each pair $m^{\prime}$ and $m^{\prime \prime}$ in $M$ there is an element $m$ in $M$ such that $m \leqq m^{\prime}$ and $m \leqq m^{\prime \prime}$. An ideal $U$ in $X$ is, as usual, a subspace such that if $a$ and $b$ are in $U$ and $a \leqq x \leqq b$ then $x$ is in $U$.

We will say an ideal $U$ is irreducible if there is an element $p$ in $U \cap P^{*}$ satisfying the following property: If $J$ is an ideal in $U$ containing $p$ and $L$ is any other ideal in $U$ containing a member of $P^{*}$ then $P \cap J \cap L \neq\{0\}$. Such an element $p$ will be referred to as an element of irreducibility. An element $w$ of $X$ will be called quasi-negative with respect to an ideal $V$ if $-w+\left(P^{*} \cap V\right) \subseteq P^{*}$.

Definition 1. Let $\left\{x_{\alpha}\right\}$ be a net in $X$. If there exists an irreducible ideal $V$ containing a set $M$ directed downward such that

1. for each $m$ in $M,-m \leqq x_{\alpha} \leqq m$ for all $\alpha$ greater than or equal to some $\alpha^{\prime}$, and

2. $t \leqq m$ for all $m$ in $M$ implies $t$ is quasi-negative with respect to $V$, then $\left\{x_{\alpha}\right\}$ is said to locally order converge (lo converge) to zero (with respect to $V$ ).

Definition 2. A net $\left\{x_{\alpha}\right\}$ in $X$ unbounded locally order converges (ulo converges) to zero if there is an irreducible ideal $V$ in $X$ and a net $\left\{z_{\alpha}\right\} \subseteq V \cap P$ such that

1. $-z_{\alpha} \leqq x_{\alpha} \leqq z_{\alpha}$ for all $\alpha$, and

2. if $\left\{y_{\alpha}\right\}$ is a bounded net (i.e. there exist $a$ and $b$ in $X$ such that $a \leqq y_{\alpha} \leqq b$ for all $\alpha$ ) satisfying $0 \leqq y_{\alpha} \leqq z_{\alpha}$ for all $\alpha$ then $\left\{y_{\alpha}\right\}$ locally order converges with respect to $V$. 
Local order convergence and unbounded local order convergence to arbitrary points in $X$ are defined by translation.

Local order convergence is a modification of the standard concept of order convergence and unbounded local order convergence is designed to extend local order convergence to unbounded nets, the extension being analogous to the extension of order convergence to unbounded order convergence in [1].

\section{The main result.}

THEOREM. Let E be a Marinescu space. There exists a partially ordered vector space $X$ and a one-to-one linear mapping $i$ from $E$ into $X$ such that a net $\left\{x_{\alpha}\right\}$ converges to $x$ in $E$ if and only if the net $\left\{i x_{\alpha}\right\}$ ulo converges to $i x$ in $X$.

Proof. Since $E$ is a Marinescu space, it is the inductive limit (in the category of convergence vector spaces) of a collection $\mathscr{D}$ of locally convex topological vector spaces. Corresponding to each $D \in \mathscr{D}$ is the family $\theta$ of all continuous seminorms on $D$. We denote by $\sigma$ the set of these families $\theta$. Let $X_{\theta}$ be the product of factors $E \times \boldsymbol{R}$ ( $R$ the real numbers) indexed by the members of $\theta$ and let $X=E \oplus\left(\oplus_{\theta \in \sigma} X_{\theta}\right)$. For an element $\boldsymbol{x}$ in $\boldsymbol{X}$ we will use the (abbreviated) notation

$$
\boldsymbol{x}=x \bigoplus_{i=1}^{n}\left(\prod_{p \in \theta_{i}}\left(x_{i}^{p}, \lambda_{i}^{p}\right)\right),
$$

where $x$ is the entry in the component $E$ and $\prod_{p \in \theta_{i}}\left(x_{i}^{p}, \lambda_{i}^{p}\right)$ is a nonzero entry in the component $X_{\theta_{i}}(i=1, \cdots, n)$, entries in all other components $X_{\theta}$ being zero. Let $p$ be a continuous seminorm on a space $D$. If $D \supseteq D_{i}$ $(i=1, \cdots, m)$ in $\mathscr{D}$ (i.e., the inclusion map from each $D_{i}$ into $D$ is continuous) then we identify $p$ with each of its restrictions and write $p \in \bigcap_{i=1}^{n} \theta_{i}$.

We now define a positive cone $\boldsymbol{P}$ for $\boldsymbol{X}$. An element $\boldsymbol{x}$ of $\boldsymbol{X}$ is in $\boldsymbol{P}$ if either $\boldsymbol{x}=0$, or its entry in some $X_{\theta}$ is nonzero and the following are satisfied:

1. For each $X_{\theta_{i}}$ all scalars $\lambda_{i}^{p}$ are nonnegative. In addition, if $x_{i}^{p} \neq 0$ then $\lambda_{i}^{p}>0$.

2. For each $p \in \bigcap_{i=1}^{n} \theta_{i}$, the element $x+\sum_{i=1}^{n} x_{i}^{p}$ is in the linear span of $\bigcup_{i=1}^{n} D_{i}$ and $p\left(x+\sum_{i=1}^{n} x_{i}^{p}\right) \leqq \sum_{i=1}^{n} \lambda_{i}^{p}$.

It is routine to verify that $\boldsymbol{P}$ is a positive cone.

Let $i: E \rightarrow X$ be the obvious embedding which maps $x$ in $E$ to that element in $\boldsymbol{X}$ whose only nonzero entry is $x$ in the factor $E$.

I. Assume $\left\{x_{\alpha}\right\}$ converges to zero in $E$. We will show that $\left\{i x_{\alpha}\right\}$ ulo converges to zero in $\boldsymbol{X}$. Since $E$ is a Marinescu space $\left\{x_{\alpha}\right\}$ converges to zero in some subspace $D \in \mathscr{D}$. We claim that $V \equiv E \oplus X_{\theta}$ is an irreducible 
ideal in $X$, where $\theta$ is the family of all continuous seminorms on $D$. It follows readily from the positivity requirements on the scalars $\lambda_{i}^{p}$ that $V$ is an ideal. Let $J$ be any ideal in $V$ containing $\prod_{p \in \theta}(0,1)$ and let $L$ be any other ideal in $V$ containing some positive element $c \oplus\left(\prod_{p \in \theta}\left(c^{p}, \gamma^{p}\right)\right)$. We choose a $q \in \theta$ for which $\gamma^{q}>0$ and consider the element $\left(c+\left(c^{q} / 2\right), \gamma^{q} / 2\right)_{\theta}$, it being understood that all other coordinates in $X_{\theta}$ are $(0,0)$. Since this element is positive and less than both $\gamma_{p}^{q}\left[\prod_{p \in \theta}(0,1)\right]$ and $c \oplus\left(\prod_{p \in \theta}\left(c_{p}, \gamma_{p}\right)\right)$, it is in $J \cap L \cap P^{*}$.

Our next step is to exhibit a net $\left\{z_{\alpha}\right\}$ in $V \cap P$ such that the conditions of Definition 2 are satisfied. We define $z_{\alpha}=\prod_{p \in \theta}\left(0, p\left(x_{\alpha}\right)\right)$ for each $\alpha$. Then $\left\{\boldsymbol{z}_{\alpha}\right\}$ is contained in $V \cap \boldsymbol{P}$ and $-\boldsymbol{z}_{\alpha} \leqq i x_{\alpha} \leqq z_{\alpha}$. Let $\left\{\boldsymbol{y}_{\alpha}\right\}$ be any bounded net in $\boldsymbol{X}$ satisfying $0 \leqq \boldsymbol{y}_{\alpha} \leqq z_{\alpha}$ for all $\alpha$. We will show that $\left\{\boldsymbol{y}_{\alpha}\right\}$ lo converges with respect to $V$. Since $V$ is an ideal, each $y_{\alpha}$ can be written

$$
\boldsymbol{y}_{\alpha}=y_{\alpha} \oplus\left(\prod_{p \in \theta}\left(y_{\alpha}^{p}, \eta_{\alpha}^{p}\right)\right) .
$$

For each $p \in \theta$ and each $\alpha$, the quantity $\beta_{\alpha}^{p} \equiv \sup _{\alpha^{\prime} \geqq \alpha} \eta_{\alpha^{\prime}}^{p}$ is finite because $\left\{\boldsymbol{y}_{\alpha}\right\}$ is bounded. Furthermore, the net $\left\{\beta_{\alpha}^{p}\right\}$ converges to zero for each $p \in \theta$ since $\left\{p\left(x_{\alpha}\right)\right\}$ converges to zero. The collection $M$ of elements $\boldsymbol{m}_{\alpha}=\prod_{p \in \theta}\left(0,2 \beta_{\alpha}^{p}\right)$ is directed and $0<\boldsymbol{y}_{\alpha^{\prime}} \leqq \boldsymbol{m}_{\alpha}$ for all $\alpha^{\prime} \geqq \alpha$. All that remains to be verified is that given $t \leqq \boldsymbol{m}_{\alpha}$ for all $\boldsymbol{m}_{\alpha}$ in $M$, then $\boldsymbol{t}$ is quasi-negative. Let

By assumption,

$$
\boldsymbol{t}=t \bigoplus_{i=1}^{n}\left(\prod_{p \in \Phi_{i}}\left(t_{i}^{p}, \tau_{i}^{p}\right)\right) .
$$

$$
p\left(-t-\sum_{i=1}^{n} t_{i}^{p}\right) \leqq 2 \beta_{\alpha}^{p}-\sum_{i=1}^{n} \tau_{i}^{p}
$$

for all $\alpha$ and for every $p \in \theta \cap\left(\bigcap_{i=1}^{n} \Phi_{i}\right)$. Since for each $p \in \theta$ the net $\left\{\beta_{\alpha}^{p}\right\}$ converges to zero,

If

$$
p\left(-t-\sum_{i=1}^{n} t_{i}^{p}\right) \leqq-\sum_{i=1}^{n} \tau_{i}^{p} .
$$

$$
\boldsymbol{u}=u \oplus\left(\prod_{p \in \theta}\left(u^{p}, \mu^{p}\right)\right)
$$

is an element in $V \cap \boldsymbol{P}^{*}$ then

$$
\begin{aligned}
p\left[(u-t)+\left(u^{p}-\sum_{i=1}^{n} t_{i}^{p}\right)\right] & \leqq p\left(u+u^{p}\right)+p\left(-t-\sum_{i=1}^{n} t_{i}^{p}\right) \\
& \leqq \mu^{p}-\sum_{i=1}^{n} \tau_{i}^{p}
\end{aligned}
$$


for all $p$ in $\theta \cap\left(\bigcap_{i=1}^{n} \Phi_{i}\right)$. One can now verify that $u-t$ is in $P^{*}$, establishing that $t$ is quasi-negative.

II. Conversely, assume $\left\{i x_{\alpha}\right\}$ ulo converges to zero in $\boldsymbol{X}$. We will show that $\left\{x_{\alpha}\right\}$ converges to zero in the Marinescu space $E$. Let $V$ denote an irreducible ideal in $X$ and $\left\{z_{\alpha}\right\}$ a net in $V \cap P$ fulfilling the requirements of Definition 2. We will establish two key facts:

1. The ideal $V \cap P$ is contained in $E \oplus \bigoplus_{i=1}^{N} X_{\theta_{i}}$ for a finite subset $\left\{\theta_{i}\right\}_{i=1}^{N}$ of $\sigma$.

2. We write $z_{\alpha}=z_{\alpha} \bigoplus_{i=1}^{N} *\left(\prod_{p \in \theta_{i}}\left(z_{\alpha i}^{p}, \zeta_{\alpha i}^{p}\right)\right)$, adopting the convention that a starred element may have entries identically zero in some of the factors $X_{\boldsymbol{\theta}_{i}}$. The net $\left\{\sum_{i=1}^{N} \zeta_{\boldsymbol{\alpha}}^{p}\right\}$, indexed by $\alpha$, converges to zero in $\boldsymbol{R}$.

From (1) we obtain

so that

$$
i x_{\alpha} \leqq z_{\alpha} \leqq \bigoplus_{i=1}^{N} *\left(\prod_{p \in \theta_{i}}\left(0,2 \zeta_{\alpha i}^{p}\right)\right)
$$

$$
p\left(x_{\alpha}\right) \leqq 2 \sum_{i=1}^{N} \zeta_{\alpha i}^{p} \text { for } p \text { in } \bigcap_{i=1}^{N} \theta_{i} .
$$

Fact (2) then allows us to conclude that $\left\{x_{\alpha}\right\}$ converges to zero in $E$ as desired.

To prove (1), let $b$ be an element of irreducibility for $V$; there is a collection $\left\{X_{\theta_{i}}\right\}_{i=1}^{N}$ such that $b$ is in $E \oplus \bigoplus_{i=1}^{N} X_{\theta_{i}}$. Assume that there is an element $c=c \bigoplus_{i=1}^{m}\left(\prod_{p \in \Phi_{i}}\left(c_{i}^{p}, \gamma_{i}^{p}\right)\right)$ in $V \cap P$ having a nonzero entry in some factor, say $X_{\Phi_{1}}$, not in the collection $\left\{X_{\theta_{i}}\right\}_{i=1}^{N}$. We choose a seminorm $q \in \Phi_{1}$, for which $\gamma_{1}^{q}>0$ and define

$$
\boldsymbol{c}^{\prime}=\left(c+\sum_{i=1}^{m} c_{i}^{a},\left[\left(\sum_{i=1}^{m} \gamma_{i}^{q}\right) \vee q\left(c+\sum_{i=1}^{m} c_{i}^{q}\right)\right]\right)_{\Phi_{1}},
$$

it being understood that all other entries in $X_{\Phi_{1}}$ are $(0,0)$. For

$$
k>\frac{1}{\gamma_{1} q}\left[\left(\sum_{i=1}^{m} \gamma_{i}^{q}\right) \vee \cdot q\left(c+\sum_{i=1}^{m} c_{i}^{q}\right)\right]
$$

it can be verified that $0<c^{\prime}<k c$; thus $c^{\prime}$ is in $V \cap P$. The ideal generated by $c^{\prime}$ is contained in the factor $X_{\Phi_{1}}$, while the ideal generated by $b$ is contained in $E \oplus\left(\oplus_{i=1}^{N} X_{\theta_{i}}\right)$. These ideals have no positive elements in common, contradicting the irreducibility of $V$. Thus $V \cap P$ is contained in $E \oplus\left(\oplus_{i=1}^{N} X_{\theta_{i}}\right)$.

To prove (2), let $q$ be a seminorm in $\bigcap_{i=1}^{N} \theta_{i}$. Given $\alpha$, if there is a least $j$ in $\{1, \cdots, N\}$ for which $\zeta_{\alpha j}^{a}>0$, we define

$$
\boldsymbol{y}_{\alpha}=\frac{1}{1+\sum \zeta_{\alpha i}^{a}}\left\{\left(z_{\alpha}+\sum z_{\alpha i}^{a}, \zeta_{\alpha j}^{a}\right)_{\theta,} \oplus \bigoplus_{i=j+1}^{n} *\left(0, \zeta_{\alpha i}^{a}\right)_{\theta i}\right\} \text {, }
$$


adopting the notation $\sum$ to mean $\sum_{i=1}^{N}$; otherwise, we let $y_{\alpha}=0$. Then $0 \leqq y_{\alpha} \leqq z_{\alpha}$ for each $\alpha$ and $\left\{y_{\alpha}\right\}$ is bounded by $\bigoplus_{i=1}^{N}\left(\prod_{p \in \theta_{i}}(0,2)\right)$. Thus the net $\left\{\boldsymbol{y}_{\alpha}\right\}$ lo converges to zero. with respect to $V$. There is a directed set $M$ contained in $V \cap P$ which fulfills the conditions of Definition 1.

For any element $m=m \bigoplus_{i=1}^{N} *\left(\prod_{p \in \theta_{i}}\left(m_{i}^{p}, v_{i}^{p}\right)\right)$ in $M$ there is an $\alpha_{0}$ such that $y_{\alpha} \leqq m$ for all $\alpha \geqq \alpha_{0}$. From the requirement in the definition of the positive cone that scalars be nonnegative, one deduces

$$
\sum \nu_{i}^{a} \geqq \sum \zeta_{\alpha i}^{a} / 1+\sum \zeta_{\alpha i}^{a} \geqq 0 \quad\left(\alpha \geqq \alpha_{0}\right) .
$$

Thus the proof of (2) will be complete if we can show that the infimum of the set $\left\{\sum_{i=1}^{N} \nu_{i}^{q}\right\}$ of scalars corresponding to elements $m$ in $M$ is zero. Assume to the contrary that this infimum is a positive number $2 \rho$. Let $2 r_{i}(i=1, \cdots, n)$ denote the infimum of $\left\{v_{i}^{q}\right\}$ for all $m$ in $M$. We choose

$$
k=k \bigoplus_{i=1}^{N} *\left(\prod_{p \in \theta_{i}}\left(k_{i}^{p}, \varepsilon_{i}^{p}\right)\right)
$$

in $M$ with $\sum \varepsilon_{i}^{q} \leqq 3 \rho$ and define

$$
s=\bigoplus_{i \in J}\left(\frac{k+\sum k_{i}^{q}}{|J|}, r_{i}\right)_{\theta_{i}},
$$

where $J$ consists of those indices $i(i=1, \cdots, n)$ for which $r_{i} \neq 0$ and $|J|$ is the cardinality of $J$. If $\boldsymbol{m} \leqq k(m \in M)$, with the help of the inequalities $q\left(k+\sum k_{i}^{q}-m-\sum m_{i}^{q}\right) \leqq \sum \varepsilon_{i}^{q}-\sum \nu_{i}^{q} \leqq \rho \leqq \sum v_{i}^{q}-\rho \leqq \sum \nu_{i}^{q}-\sum r_{i}$

one can verify that $s \leqq m$. The fact that $M$ is directed downward implies that $s \leqq m$ for all $m$ in $M$. To reach a contradiction we show that $s$ is not quasi-negative with respect to $V$. There is an integer $1 \leqq j \leqq N$ for which $\varepsilon_{j}^{q}>0$. We choose $\omega>0$ small enough so that $\omega \sum \varepsilon_{i}^{q}<\varepsilon_{j}^{q}$, and if $r_{j} \neq 0$ we require $\omega \sum \varepsilon_{i}^{q}<r_{j}$. Defining $t=\left(k+\sum k_{i}^{q}, \sum \varepsilon_{i}^{q}\right)_{\theta_{j}}$ we observe $0<\omega t<k$ and thus $\omega t$ is in $V \cap P^{*}$. The fact that $M$ is directed downward implies that at least one $r_{i}(i=1, \cdots, N)$ is nonzero. It follows that $-s+\omega t$ is not in $\boldsymbol{P}^{*}$, completing the proof of (2).

We note that the inductive limit of a directed system of Marinescu spaces in the category of convergence vector spaces exists and is itself a Marinescu space [2]. Thus the theorem applies to inductive systems of Marinescu spaces.

\section{REFERENCES}

1. Ralph DeMarr, Partially ordered linear spaces and locally convex linear topological spaces, Illinois J. Math. 8 (1964), 601-606. MR 30 \#1388.

2. H. Jarchow, Marinescu Räume, Comment. Math. Helv. 44 (1969), 138-163. MR 40 \#3260. 
3. G. Marinescu, Espaces vectoriels pseudotopologiques et théorie des distributions, Họchschulbücher für Math., Band 59, VEB Deutscher Verlag der Wissenschaften, Berlin, 1963. MR 29 \#3878.

4. A. L. Peressini, Ordered topological vector spaces, Harper \& Row, New York, 1967. MR 37 \#3315.

Department of Mathematics, University of Arkansas, Fayetteville, Arkansas 72701 